\title{
CORRECTION
}

\section{Correction to: Focus on ventilation and ARDS: recent insights}

\author{
Audrey De Jong ${ }^{1 *}$ (D, Samir Jaber ${ }^{1}$ and Niall D. Ferguson ${ }^{2,3,4}$
}

(0) 2019 Springer-Verlag GmbH Germany, part of Springer Nature

\section{Correction to: Intensive Care Med (2019) 45:1635-1638 https://doi.org/10.1007/s00134-019-05804-w}

The original version of this article unfortunately contained a mistake. There was an error in the title. The correct title reads: Focus on ventilation and ARDS: recent insights.

We apologise for the mistake.

\section{Author details}

1 Department of Anesthesia and Intensive Care Unit, Regional University Hospital of Montpellier, PhyMedExp, University of Montpellier, INSERM U1046, CNRS UMR, 9214, St-Eloi Hospital, Montpellier, France. ${ }^{2}$ Interdepartmental Division of Critical Care Medicine, Departments of Medicine and Physiology and Institute of Health Policy, Management and Evaluation, University of Toronto, Toronto, Canada. ${ }^{3}$ Division of Respirology, Department of Medicine, University Health Network and Sinai Health System, 585 University Avenue, PMB 11-120, Toronto, ON M5G 2N2, Canada. ${ }^{4}$ Toronto General Hospital Research Institute, Toronto, Canada.

Published online: 30 October 2019

\footnotetext{
*Correspondence: a-de_jong@chu-montpellier.fr

1 Department of Anesthesia and Intensive Care Unit, Regional University Hospital of Montpellier, PhyMedExp, University of Montpellier, INSERM

U1046, CNRS UMR, 9214, St-Eloi Hospital, Montpellier, France

Full author information is available at the end of the article

\section{Springer}

